\title{
Some Remarks on Glaisher-Ramanujan Type Integrals
}

\author{
Alexander E. Patkowski ${ }^{1}$, Marek Wolf ${ }^{2}$ \\ ${ }^{1} 1390$ Bumps River Rd. Centerville, MA 02632, USA \\ E-mail: alexpatk@hotmail.com \\ ${ }^{2}$ Cardinal Stefan Wyszynski University \\ Faculty of Mathematics and Natural Sciences. College of Sciences \\ ul. Wóycickiego 1/3, Auditorium Maximum, (room 113) \\ PL-01-938 Warsaw, Poland \\ E-mail:m.wolf@uksw.edu.pl
}

Received: 06 February 2016; revised: 23 May 2016 ; accepted: 24 May 2016 ; published online: 08 June 2016

\begin{abstract}
Some integrals of the Glaisher-Ramanujan type are established in a more general form than in previous studies. As an application we prove some Ramanujan-type series identities, as well as a new formula for the Dirichlet beta function at the value $s=3$. We also present a Mathematica program calculating values of beta function at odd positive arguments.
\end{abstract}

Key words: Fourier integrals, Dedekind eta function, trigonometric series

\section{INTRODUCTION}

In the paper by Glasser [1], the work of Glaisher [2] and Ramanujan [3] was extended to present further evaluations of the integral

$$
\int_{0}^{\infty} \eta^{n}(i x) f(x) d x
$$

for integers $n \geq 1$, and particular elementary functions $f(x)$. Here, as usual, the Dedekind-eta function is given by

$$
\eta(i x)=q^{1 / 24} \prod_{n \geq 1}^{\infty}\left(1-q^{n}\right),
$$

where $q=e^{-2 \pi x}$, for real $x>0$. For some commentary on integrals of this type we refer the reader to $[1,3]$ and references therein.

In this note we restrict our attention to the case $f(x)=$ $e^{-b^{2} x} \cos (c x)$, and $f(x)=e^{-b^{2} x} \sin (c x)$, in (I.1). This provides a refinement to integrals like (8) of [1] and (19)-(28) in [2]. That is, we shall prove:

Theorem I. 1 For $b>0$ and $c>0$ we have

$$
\begin{aligned}
& \int_{0}^{\infty} \eta^{3}(i 4 x / \pi) e^{-b^{2} x} \sin (c x) d x= \\
= & \frac{\pi}{4} \frac{\sinh \left(\frac{\pi}{2} A(b, c)\right) \sin \left(\frac{\pi}{2} B(b, c)\right)}{\sinh ^{2}\left(\frac{\pi}{2} A(b, c)\right)+\cos ^{2}\left(\frac{\pi}{2} B(b, c)\right)},
\end{aligned}
$$

$$
\begin{aligned}
& \int_{0}^{\infty} \eta^{3}(i 4 x / \pi) e^{-b^{2} x} \cos (c x) d x= \\
= & \frac{\pi}{4} \frac{\cosh \left(\frac{\pi}{2} A(b, c)\right) \cos \left(\frac{\pi}{2} B(b, c)\right)}{\cosh ^{2}\left(\frac{\pi}{2} A(b, c)\right)-\sin ^{2}\left(\frac{\pi}{2} B(b, c)\right)},
\end{aligned}
$$

where $2 A(b, c)^{2}=\sqrt{b^{4}+c^{2}}+b^{2}$, and $2 B(b, c)^{2}=$ $\sqrt{b^{4}+c^{2}}-b^{2}$.

Note that $c \rightarrow 0$ of (I.4) gives (14) of [1]:

$$
\int_{0}^{\infty} \eta^{3}(i 4 x / \pi) e^{-y x} d x=\frac{1}{\cosh (\sqrt{\pi y})}
$$

Formally integrals (I.3) and (I.4) can be obtained from the results of Glasser inserting $b^{2}+i c$ instead of $b^{2}$.

From here we can easily extend the work of Glasser to obtain evaluations of integrals involving $\eta^{n}(i a x) \eta^{k}(i b x)$, for integrals $n, k \geq 1$, and $a, b \in \mathbb{R}$. Throughout this paper we define

$$
\chi(n)=\left\{\begin{array}{lll}
0, & \text { if } n=0 & (\bmod 2), \\
1, & \text { if } n=1 \quad(\bmod 4), \\
-1, & \text { if } n=3 & (\bmod 4) .
\end{array}\right.
$$


Theorem I. 2 For $c>0$ we have

$$
\begin{aligned}
& \int_{0}^{\infty} \eta^{6}(i 4 x / \pi) \sin (c x) d x= \\
= & \frac{\pi}{4} \sum_{n \geq 1} \chi(n) n \frac{\sinh \left(\frac{\pi}{2} A(n, c)\right) \sin \left(\frac{\pi}{2} B(n, c)\right)}{\sinh ^{2}\left(\frac{\pi}{2} A(n, c)\right)+\cos ^{2}\left(\frac{\pi}{2} B(n, c)\right)}, \\
& \int_{0}^{\infty} \eta^{6}(i 4 x / \pi) \cos (c x) d x= \\
= & \frac{\pi}{4} \sum_{n \geq 1} \chi(n) n \frac{\cosh \left(\frac{\pi}{2} A(n, c)\right) \cos \left(\frac{\pi}{2} B(n, c)\right)}{\cosh ^{2}\left(\frac{\pi}{2} A(n, c)\right)-\sin ^{2}\left(\frac{\pi}{2} B(n, c)\right)} .
\end{aligned}
$$

Unfortunately, as we observe, the right sides of (I.4) and (I.5) are not expressible in terms of elementary functions like those of Glasser's [1] and Glaisher's [2]. However the terms in the series on rhs of eqs. (I.6), (I.7) are quickly decreasing with $n$. We can obtain two types of approximations: in one form we keep only a finite number of terms in the series, in the second approximation we skip in the denominators the terms $\sin ^{2}\left(\frac{\pi}{2} B(n, c)\right)$ and $\cos ^{2}\left(\frac{\pi}{2} B(n, c)\right)$ in comparison with hyperbolic sinus and hyperbolic cosinus leaving the sum of terms $\chi(n) n \sin \left(\frac{\pi}{2} B(n, c)\right) / \sinh \left(\left(\frac{\pi}{2} A(n, c)\right)\right.$ and $\chi(n) n \cos \left(\frac{\pi}{2} B(n, c)\right) / \cosh \left(\left(\frac{\pi}{2} A(n, c)\right)\right.$ respectively. Because $\sinh (x) \approx \exp (x) / 2$ for large $x$ thus in the infinite sum only several first terms can be maintained giving practically closed expressions for integrals on lhs of eqs. (I.6) and (I.7):

$$
\begin{aligned}
& \int_{0}^{\infty} \eta^{6}(i 4 x / \pi) \sin (c x) d x \approx \\
\approx & \frac{\pi}{4}\left(\frac{\sin \left(\frac{\pi}{2} B(1, c)\right)}{\sinh \left(\frac{\pi}{2} A(1, c)\right)}-3 \frac{\sin \left(\frac{\pi}{2} B(3, c)\right)}{\sinh \left(\frac{\pi}{2} A(3, c)\right)}+\ldots\right) \\
& \int_{0}^{\infty} \eta^{6}(i 4 x / \pi) \cos (c x) d x \approx \\
\approx & \frac{\pi}{4}\left(\frac{\cos \left(\frac{\pi}{2} B(1, c)\right)}{\cosh \left(\frac{\pi}{2} A(1, c)\right)}-3 \frac{\cos \left(\frac{\pi}{2} B(3, c)\right)}{\cosh \left(\frac{\pi}{2} A(3, c)\right)}+\ldots\right)
\end{aligned}
$$

We have checked numerically that these approximations are already very accurate for a few first terms. We have used the free package PARI/GP [4]. It has a built-in procedure for calculating the $\eta$ function as well as the procedure for numerical integration. Because $\eta$ is not defined for 0 we have used as the lower limit of integration on lhs in (I.6) and (I.7) the value $x=0.00000001$ and we have checked that taking for example $x=0.00001$ produces practically the same numbers. In Figures 1-4 we present the accuracy of such approximations for $c \in(0,20)$, for $c \rightarrow \infty$ the integrals and series on both sides of (I.6) and (I.7) tend to zero. Surprisingly, such simplifications very accurately reproduce values of integrals, thus in fact we can say we have closed forms for them.

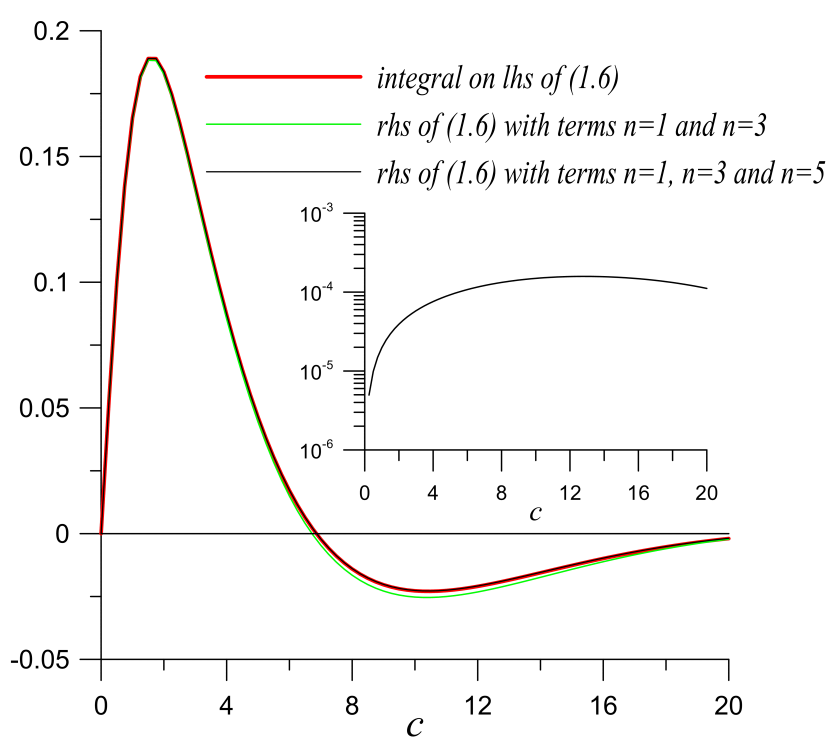

Fig. 1. The plot of lhs of (I.6) compared with the sum of terms $n=3$ and $n=5$ on rhs of (I.6). In the inset the plot of the absolute value of the difference of the integral and approximation for $n=5$ is presented on the logarithmic $y$-axis

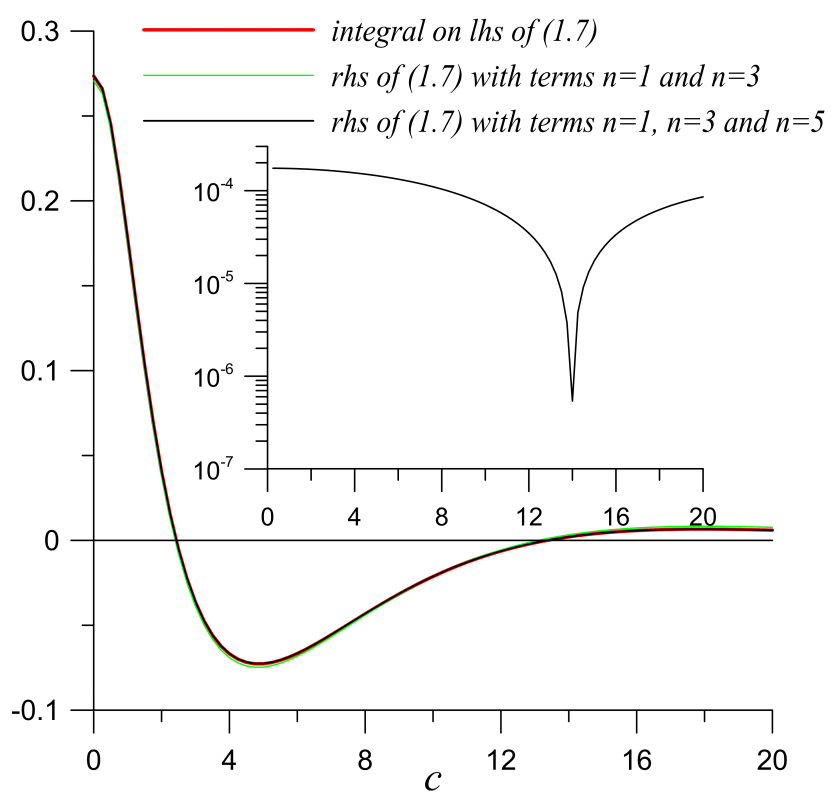

Fig. 2. The plot of lhs of (I.7) compared with the sum of terms $n=3$ and $n=5$ on rhs of (I.7). In the inset the plot of the absolute value of the difference of the integral and approximation for $n=5$ is presented on the logarithmic $y$-axis. The cusp around $c=14$ is due to the change of sign of the difference

We can also obtain other examples using the same procedure as Glasser, by appealing to different theta series and using Theorem I.1. In particular, by Euler's identity [5] we have the following: 
Theorem I. 3 For $c>0$ we have

$$
\begin{aligned}
& \int_{0}^{\infty} \eta^{3}(i 4 x / \pi) \eta(i 12 x / \pi) \sin (c x) d x=\frac{\pi}{4} \sum_{n \in \mathbb{Z}}(-1)^{n} \\
\times & \frac{\cosh \left(\frac{\pi}{2} A(6 n+1, c)\right) \cos \left(\frac{\pi}{2} B(6 n+1, c)\right)}{\cosh ^{2}\left(\frac{\pi}{2} A(6 n+1, c)\right)-\sin ^{2}\left(\frac{\pi}{2} B(6 n+1, c)\right)} .
\end{aligned}
$$

Again we can approximate the above series by following terms:

$$
\begin{aligned}
& \int_{0}^{\infty} \eta^{3}(i 4 x / \pi) \eta(i 12 x / \pi) \sin (c x) d x \approx \\
\approx & \frac{\pi}{4}\left(-\frac{\cos \left(\frac{\pi}{2} B(5, c)\right)}{\cosh \left(\frac{\pi}{2} A(5, c)\right)}+\frac{\cos \left(\frac{\pi}{2} B(1, c)\right)}{\cosh \left(\frac{\pi}{2} A(1, c)\right)}\right. \\
- & \left.\frac{\cos \left(\frac{\pi}{2} B(7, c)\right)}{\cosh \left(\frac{\pi}{2} A(7, c)\right)}\right)
\end{aligned}
$$

The single middle term above already gives the values of the integral on lhs of (I.10) with a few percent accuracy, see Fig. 5. Both sides of (I.10) tend to zero when $c \rightarrow \infty$.

\section{THE PROOF}

To prove Theorem I.1 we require some simple series evaluations that we were unable to find in the literature. Our methods are similar to those of [2] and we only require some known integral evaluations and the Poisson summation formula for Fourier sine transforms [6, p.257]. If $f(x)$ is a continuous, real-valued function with bounded total variation on $[a, b]$ then

$$
\sum_{a \leq n \leq b} \chi(n) f(n)=\sum_{n \geq 1} \chi(n) \int_{a}^{b} f(x) \sin (\pi x n / 2) d x .
$$

By I. S. Gradshteyn and I. M. Ryzhik [7, p.428], we have

$$
\begin{gathered}
\int_{0}^{\infty} \frac{x \sin (a x) d x}{\left(x^{2}+b^{2}\right)^{2}+c^{2}}=\frac{\pi}{2 c} e^{-a A(b, c)} \sin (a B(b, c)), \\
\int_{0}^{\infty} \frac{x\left(x^{2}+b^{2}\right) \sin (a x) d x}{\left(x^{2}+b^{2}\right)^{2}+c^{2}}=\frac{\pi}{2} e^{-a A(b, c)} \cos (a B(b, c)),
\end{gathered}
$$

where $A(b, c)$ and $B(b, c)$ are as in Theorem I.1, and $a>0$, $b>0$, and $c>0$.

Lemma II. 4 For $b>0$ and $c>0$ we have

$$
\begin{aligned}
& \sum_{n \geq 1} \chi(n) \frac{n}{\left(n^{2}+b^{2}\right)^{2}+c^{2}}= \\
= & \frac{\pi}{4 c} \frac{\sinh \left(\frac{\pi}{2} A(b, c)\right) \sin \left(\frac{\pi}{2} B(b, c)\right)}{\sinh ^{2}\left(\frac{\pi}{2} A(b, c)\right)+\cos ^{2}\left(\frac{\pi}{2} B(b, c)\right)},
\end{aligned}
$$

$$
\begin{aligned}
& \sum_{n \geq 1} \chi(n) \frac{n\left(n^{2}+b^{2}\right)}{\left(n^{2}+b^{2}\right)^{2}+c^{2}}= \\
= & \frac{\pi}{4} \frac{\cosh \left(\frac{\pi}{2} A(b, c)\right) \cos \left(\frac{\pi}{2} B(b, c)\right)}{\cosh ^{2}\left(\frac{\pi}{2} A(b, c)\right)-\sin ^{2}\left(\frac{\pi}{2} B(b, c)\right)} .
\end{aligned}
$$

Proof. For (II.4) apply (II.1) with $f(x)=\frac{x}{\left(x^{2}+b^{2}\right)^{2}+c^{2}}$ and invoke (II.2). For (II.5) apply (II.1) with $f(x)=\frac{x\left(x^{2}+b^{2}\right)}{\left(x^{2}+b^{2}\right)^{2}+c^{2}}$ and invoke (II.3).

For (II.3), we use identity (12) of [1] (with $x$ replaced by $x 4 / \pi$ ) to find, by absolute convergence,

$$
\begin{gathered}
\int_{0}^{\infty} \eta^{3}(i x 4 / \pi) e^{-b^{2} x} \sin (c x) d x \\
=\sum_{n \geq 1} \chi(n) n \int_{0}^{\infty} e^{-\left(b^{2}+n^{2}\right) x} \sin (c x) d x \\
=c \sum_{n \geq 1} \chi(n) \frac{n}{\left(n^{2}+b^{2}\right)^{2}+c^{2}} .
\end{gathered}
$$

By (II.4) of Lemma II.1 the proof is straightforward. It is not difficult to prove (II.4). The only difference is that we appeal to the Fourier cosine transform and employ (II.5).

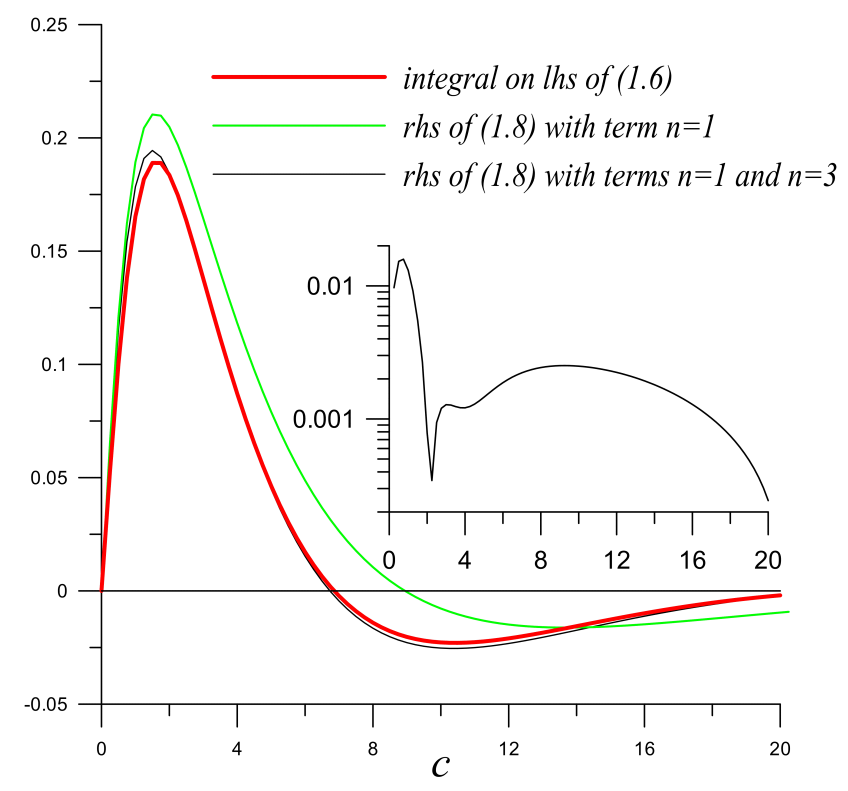

Fig. 3. The plot of lhs of (I.6) compared with the sum of terms $n=1$ and $n=3$ on rhs of (I.8). In the inset the plot of the absolute value of the difference of the integral and approximation for $n=3$ is presented on the logarithmic $y$-axis 


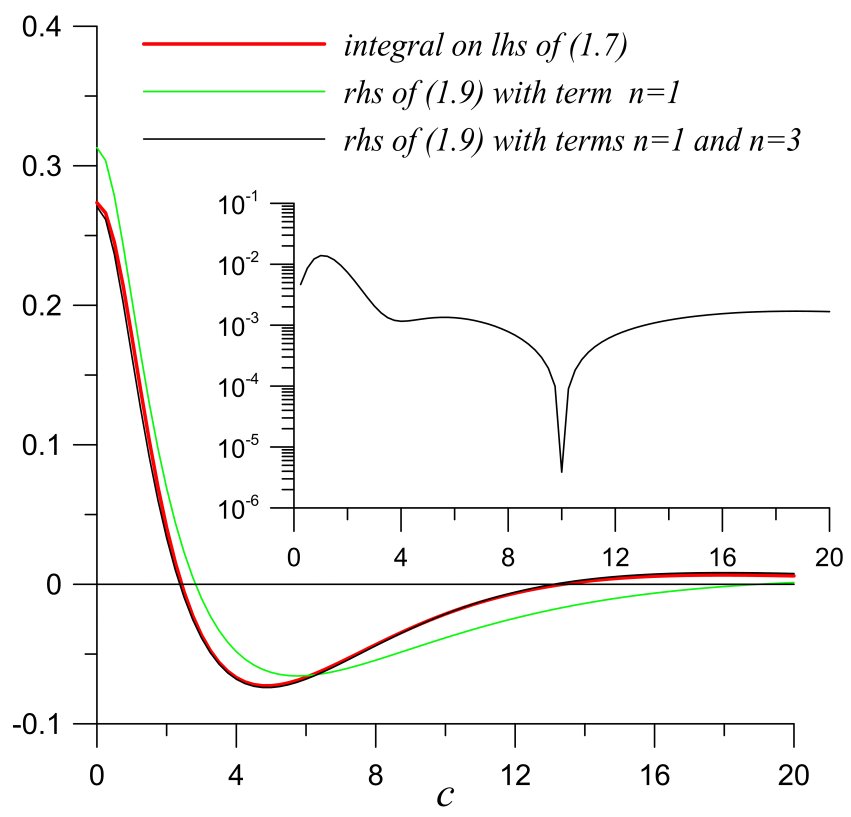

Fig. 4. The plot of lhs of (I.7) compared with the sum of terms $n=1$ and $n=3$ on rhs of (I.9). In the inset the plot of the absolute value of the difference of the integral and approximation for $n=3$ is presented on the logarithmic $y$-axis. The cusp around $c=10$ is due to the change of sign of the difference

\section{APPLICATION TO RAMANUJAN-TYPE SERIES}

In Ramanujan's notebook [6, pg. 314, eq.(8.3)] we find the amazing formula for $\zeta\left(\frac{1}{2}\right):$ If $x>0$, then

$$
\begin{aligned}
& \sum_{n \geq 1}^{\infty} \frac{1}{e^{n^{2} x}-1}=\frac{\pi^{2}}{6 x}+\frac{1}{2} \sqrt{\frac{\pi}{x}} \zeta\left(\frac{1}{2}\right)+\frac{1}{4}+\sqrt{\frac{\pi}{2 x}} \\
\times & \sum_{n \geq 1} \frac{1}{\sqrt{n}}\left(\frac{\cos \left(\frac{\pi}{4}+2 \pi \sqrt{\pi n / x}\right)-e^{-2 \pi \sqrt{\pi n / x}} \cos \left(\frac{\pi}{4}\right)}{\cosh (2 \pi \sqrt{\pi n / x})-\cos (2 \pi \sqrt{\pi n / x})}\right) .
\end{aligned}
$$

Several authors have produced generalizations of this formula $[6,8,9,10]$. Author [10] obtains a formula for the Dirichlet $L$-function for $\bar{\chi}$, the primitive Dirichlet character modulo $q$, at $s=1$. In this section we will obtain a formula for the special value $s=3$ of the Dirichlet beta function [5]

$$
\beta(s)=\sum_{n \geq 0} \frac{(-1)^{n}}{(2 n+1)^{s}} .
$$

The values of $\beta(s)$ are known, among others, for any positive odd integer:

$$
\beta(2 k+1)=\frac{(-1)^{k} E_{2 k} \pi^{2 k+1}}{4^{k+1}(2 k) !},
$$

where $E_{n}$ are the Euler numbers. In particular we have

$$
\beta(3)=\frac{\pi^{3}}{32}=0.9689461462 \ldots .
$$

In the Appendix we present a short Mathematica script calculating values of $\beta(2 k+1)$ based on the algorithm invented by D. Shanks and J. Wrench Jr. in [11].
We will prove:

Theorem III. 5 For $z>0$ we have

$$
\begin{gathered}
\frac{\pi}{8}+\sum_{n \geq 1} \frac{\chi(n)}{n\left(e^{n^{2} z}-1\right)} \\
=\frac{\beta(3)}{z}+\frac{1}{2} \sum_{n \geq 1} \frac{\sinh \left(\frac{\pi}{2} \sqrt{\frac{n \pi}{z}}\right) \sin \left(\frac{\pi}{2} \sqrt{\frac{n \pi}{z}}\right)}{n\left(\cosh \left(\pi \sqrt{\frac{n \pi}{z}}\right)+\cos \left(\pi \sqrt{\frac{n \pi}{z}}\right)\right)} .
\end{gathered}
$$

Proof. Under the same hypothesis as for sine transforms for the Poisson summation formula, we have [6, p.252]

$$
\sum_{a \leq n \leq b}^{\prime} f(n)=\int_{a}^{b} f(x) d x+2 \sum_{n \geq 1} \int_{a}^{b} f(x) \cos (\pi 2 x n) d x
$$

with the additional condition that the prime on the sum indicates only $\frac{1}{2} f(a)$ is counted if $a$ is finite, and similarly for $b$. We choose the function $(x, z>0)$

$$
f(x)=\sum_{k \geq 1} \chi(k) \frac{e^{-k^{2} x z}}{k} .
$$

Because terms in the series above tend very quickly to zero for $x>1$ only the first term in (III.21) is significant, see Fig. 6.

Glaisher [2, eq.(23)] offers

$$
\begin{aligned}
& \sum_{n \geq 1} \chi(n) \frac{e^{-n^{2} z}}{n}= \\
= & \frac{1}{2} \int_{0}^{\infty} \cos (z x) \frac{\sinh \left(\frac{\pi}{2} \sqrt{\frac{x}{2}}\right) \sin \left(\frac{\pi}{2} \sqrt{\frac{x}{2}}\right) d x}{x\left(\cosh \left(\pi \sqrt{\frac{x}{2}}\right)+\cos \left(\pi \sqrt{\frac{x}{2}}\right)\right)},
\end{aligned}
$$

or, by absolute convergence,

$$
\begin{aligned}
& \int_{0}^{\infty} \cos (z x) \sum_{n \geq 1} \chi(n) \frac{e^{-n^{2} z \alpha}}{n} d z= \\
= & \frac{1}{2} \frac{\sinh \left(\frac{\pi}{2} \sqrt{\frac{x}{2 \alpha}}\right) \sin \left(\frac{\pi}{2} \sqrt{\frac{x}{2 \alpha}}\right)}{x\left(\cosh \left(\pi \sqrt{\frac{x}{2 \alpha}}\right)+\cos \left(\pi \sqrt{\frac{x}{2 \alpha}}\right)\right)},
\end{aligned}
$$

for $\alpha>0$. Choosing $a=0$ and $b=\infty$ in (III.4) with our choice of $f(x)$, we get the theorem after noting

$$
\int_{0}^{\infty} f(x) d x=\frac{\beta(3)}{z}
$$

and $f(0)=\frac{\pi}{4}$. 


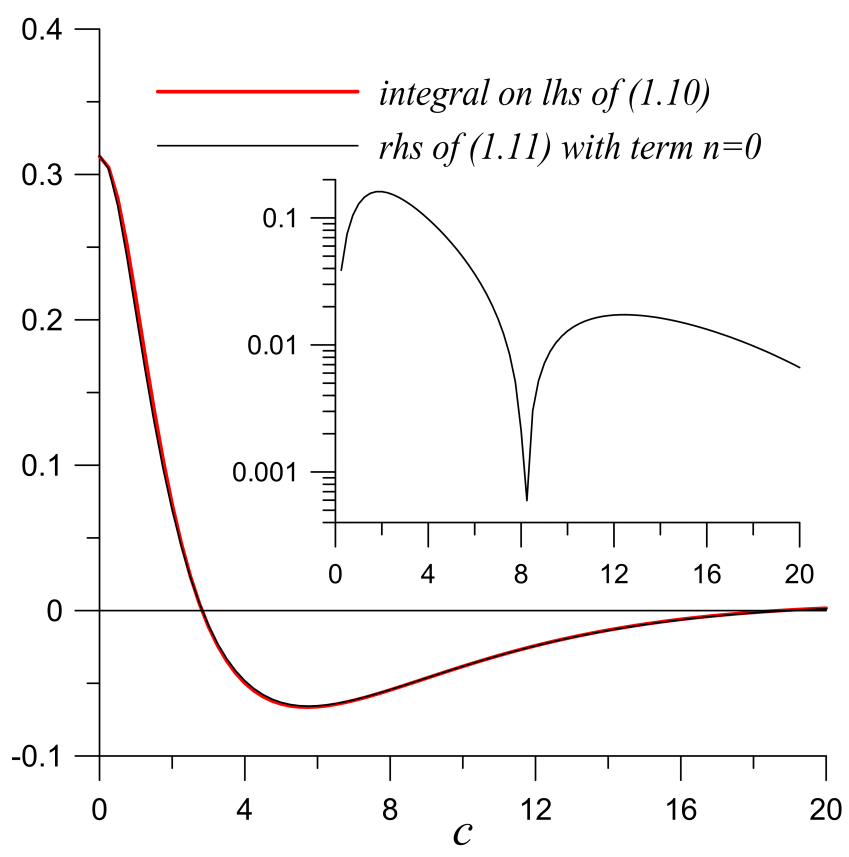

Fig. 5. The plot of lhs of (I.10) compared with the (largest) middle term in (I.11) corresponding to $n=0$. In the inset the plot of the absolute value of the difference of the integral and approximation is presented on the logarithmic $y$-axis. The cusp around $c=8$ is due to the change of sign of the difference in (I.10)

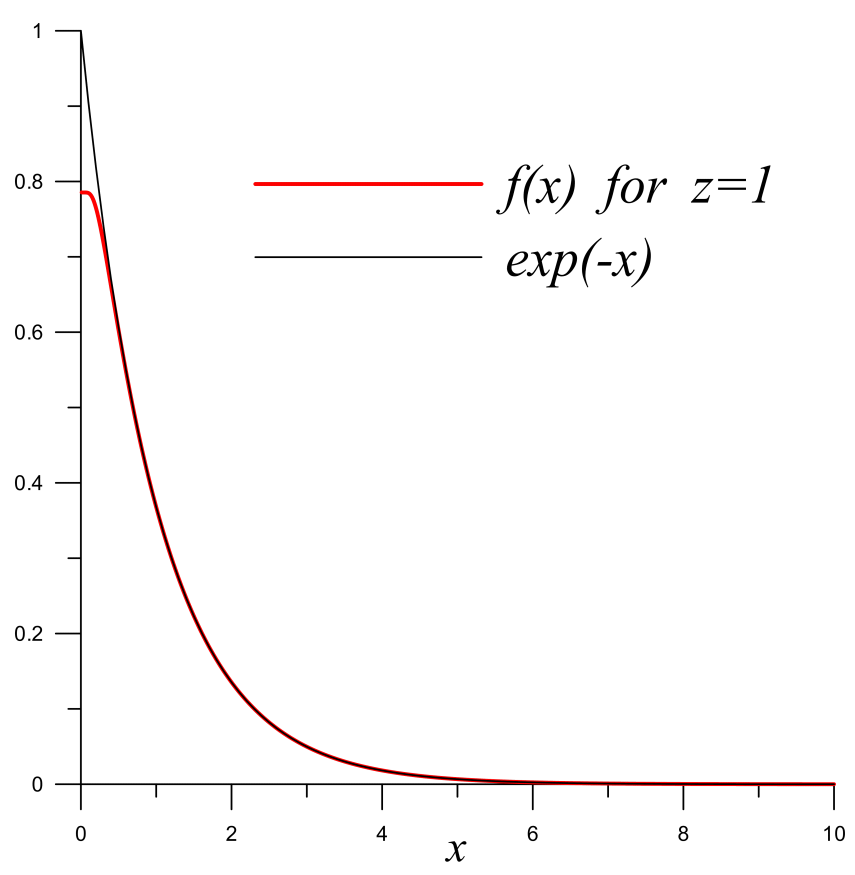

Fig. 6. The plot of function (III.21) for $z=1$ compared with exponential $e^{-x}$

\section{Appendix}

In the Fig. 7 we present the short Mathematica program based on the algorithm presented in [11] and below in Tab. 1 some values of $\beta(2 k+1)$ obtained by using this script.

$\mathrm{SS}\left[\mathrm{x}_{-}, 1\right]=\mathrm{Pi} / 4$

constantofintegration $=0$;

For $[\mathrm{k}=1, \mathrm{k}<101, \mathrm{k}++$,

tmp $=\mathrm{ss}[1 / 4, \mathrm{k}] / /$ Fullsimplify

Print [" \$\$ L_1 1 ", $2 * \mathrm{k}-1, ")="$, TeXForm [ tmp] , " $\$ \$ "]$;

$\mathrm{CC}\left[\mathrm{x}_{-}, \mathrm{k}\right]=-2 * \mathrm{Pi} *$ Integrate $[\mathrm{SS}[\mathrm{x}, \mathrm{k}], \mathrm{x}]$;

constantofintegration $=-(\operatorname{CC}[0, \mathrm{k}]+\mathrm{CC}[1 / 2, \mathrm{k}]) / 2$

$\operatorname{SS}\left[\mathrm{x}_{-}, \mathrm{k}+1\right]=2 * \mathrm{Pi} *$ Integrate $[\mathrm{CC}[\mathrm{u}, \mathrm{k}]+$ constantofintegration, $\left.\{\mathrm{u}, 0, \mathrm{x}\}]\right]$

Fig. 7. The script producing the $\mathrm{LT}_{\mathrm{E}} \mathrm{X}$ file with values of $\beta(2 k+1)$

Tab. 1. Values of $\beta(2 k+1)$

$$
\begin{aligned}
& \beta(1)=\frac{\pi}{4} \\
& \beta(3)=\frac{\pi^{3}}{32} \\
& \beta(5)=\frac{5 \pi^{5}}{1536} \\
& \beta(7)=\frac{61 \pi^{7}}{184320} \\
& \beta(9)=\frac{277 \pi^{9}}{8257536} \\
& \beta(11)=\frac{50521 \pi^{11}}{14863568} \\
& \beta(13)=\frac{540553 \pi^{13}}{1569592442880} \\
& \beta(15)=\frac{1569592442880}{199360981 \pi^{15}} \\
& \begin{array}{c}
\beta(15)=\frac{199360981 \pi}{5713316492083200} \\
\beta(17)=\frac{3878302429 \pi^{17}}{109695676479974400}
\end{array} \\
& \beta(17)=\frac{3878302429 \pi^{1}}{1096956766479974400} \\
& \beta(19)=\frac{2404879675441 \pi^{19}}{6713375410857443328000} \\
& \beta(21)=\frac{14814847529501 \pi^{21}}{408173224980132554342400} \\
& \beta(23)=\frac{69348874393137901 \pi^{23}}{1885760299408212401061888000} \\
& \beta(25)=\frac{238685140977801337 \pi^{2}}{640578267860512766391484416000} \\
& \beta(27)=\frac{4087072509293123892361 \pi^{27}}{108257727268426567520160866040400000} \\
& \beta(29)=\frac{13181680435827682794403 \pi^{29}}{3446014392207602235168067996876800000} \\
& \beta(31)=\frac{441543893249023104553682821 \pi^{31}}{113925235806383329894656327976747008000000} \\
& \beta(33)=\frac{2088463430347521052196056349 \pi^{33}}{53182980668203418002587801107262603264000000} \\
& \beta(35)=\frac{80723299235887898062168247453281 \pi^{35}}{20288243465306239899627194366398537893150720000000} \\
& \beta(37)=\frac{634185543684887724959185841096293 \pi^{37}}{1573119185617591524524939378563825092022763520000000}
\end{aligned}
$$

\section{Acknowledgments}

We thank Prof. K. Wojciechowski and an anonymous referee for their useful suggestions and remarks.

\section{References}

[1] M.L. Glasser, Some integrals of the Dedekind $\eta$-function, J. Math. Anal. Appl. 354 490-493 (2009).

[2] J.W.L. Glaisher, On the summation by definite integrals of geometric series of the second and higher order, The Quarterly Journal of Pure and Applied Mathematics, 328-343 (1871).

[3] G.H. Hardy, Ramanujan, Cambridge University Press, 1940.

[4] The PARI Group, Bordeaux.PARI/GP, version 2 . 3 . 2, 2008, available from http://pari.math.u-bordeaux.fr/. 
[5] G. Andrews, R. Askey, and R. Roy. Special Functions, volume 71 of Encyclopedia of Mathematics and its Applications. Cambridge University Press, New York, 1999.

[6] B.C. Berndt, Ramanujans Notebooks, Part II , Springer, 1989.

[7] I.S. Gradshteyn and I.M. Ryzhik. Table of Integrals, Series, and Products. Edited by A.Jeffrey and D. Zwillinger. Academic Press, New York, 7th edition, 2007.

[8] B.C. Berndt and R.J. Evans, Chapter 15 of the Ramanujan Second Notebook : Part II, Modular forms, Acta Arith. 47, 123-142 (1986).
[9] S. Kanemitsu, Y. Tanigawa, M. Toshimoto, On the values of the Riemann zeta-function at rational arguments, HardyRamanujan J. 24 10-18, 2001.

[10] D. Klusch, On Entry 8 of Chapter 15 of Ramanujan's Notebook II, Acta Arith. 58 59-64, 1991.

[11] D. Shanks and J. Wrench Jr, The Calculation of Certain Dirichlet Series, Math. of Comput. 17, 136-154 (1963).

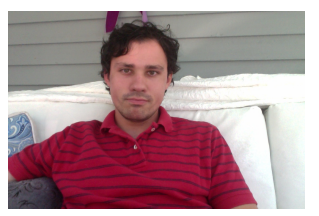

Alexander Patkowski was born in Massachusetts, USA in 1984. He is currently an Associate working in finance at an investment bank in Boston. He obtained a BS in Mathematics from Worcester Polytechnic Institute in 2008, and a Certificate in Accounting from Northeastern University in 2012. He has also taught calculus at the University of Florida from 2008 to 2009, and University of Regina in 2010. His research interests include Special Functions and Number Theory. He enjoys traveling to Europe, and has lived in Zurich (Switzerland), London (UK), and Warsaw (Poland).

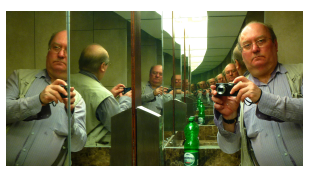

Marek Wolf obtained PhD degree in physics (1982) and habilitation (1993) at the Wroclaw University, where he was employed till 2011. Since 1984 he has been doing computer experiments in physics and mathematics. In 1991 and 1993 he was a research fellow at the Center for Polymer Studies at the Boston University. Since 2011 he is a member of the Faculty of Mathematics and Natural Sciences, College of Sciences at the Cardinal Stefan Wyszynski University in Warsaw. His hobby is photography. 